\title{
Emission of Thermal Photon in Heavy-Ion Collisions
}

\author{
Poonam Jain1, Yogesh Kumar1, Deepak Kumar² \\ ${ }^{1}$ Department of Physics, Sri Aurobindo College, University of Delhi, New Delhi, India \\ ${ }^{2}$ Department of Physics, Shyam Lal College, University of Delhi, New Delhi, India \\ Email: poonam.jn1@gmail.com, yogesh.du81@gmail.com
}

Received 1 April 2014; revised 3 May 2014; accepted 1 June 2014

Copyright (C) 2014 by authors and Scientific Research Publishing Inc.

This work is licensed under the Creative Commons Attribution International License (CC BY).

http://creativecommons.org/licenses/by/4.0/

(c) (i) Open Access

\begin{abstract}
We study the leading order processes for photon production using a phenomenological model of quark-gluon plasma (QGP) in relativistic heavy-ion collisions incorporating the parametrization factors in thermal dependent quark mass. The measurement of photon emission provides valuable insights into the early conditions of QGP. The production rate is observed in the low and intermediate range of energy and transverse momentum. The photon yield is found to increase marginally with the effect of thermal dependent quark mass. The results are little enhanced and in good agreement with other work.
\end{abstract}

\section{Keywords}

\section{Photons, Quark-Gluon Plasma}

\section{Introduction}

Many interesting outcomes have been observed at RHIC and LHC where a hot and dense matter is expected to be created. From low to high energy experiments, extensive effort have been made to probe the properties of the quark-gluon plasma (QGP), which has become a new worth of quantum cromo-dynamics (QCD) matter in the study of relativistic heavy-ion collisions [1]-[29]. First possibility for the discovery of the QGP in these experiments have been reported [30]-[32]. Photons and leptons are electromagnetic probes which are created during the evolution of a nuclear collision. In heavy-ion collisions, photons are taken as good messenger to investigate properties of the matter since they leave the medium without a strong interaction once they are produced.

The study of photon is especially interesting because they emit in the course of a heavy-ion reaction which is considered as a good observable for the space-time evolution of the colliding system. The photons are emitted 
from every stage of the collisions, and their transverse momentum are characterized by their origins. Especially, the low and intermediate transverse momentum region is considered as a suitable window for measuring the medium-induced direct photons. The high-energy photons are sensitive observables to the dynamics of the deconfined phase. Since long, Shuryak proposed photons as a promising direct probe [33] and other groups continue to be actively investigated [34]-[53]. Unfortunately, most of the photons measured in heavy-ion collisions come from hadronic decays. The experimental challenge of obtaining spectra of direct photons has been gone through by several experiments; WA98 at SPS/CERN [54]-[56] and PHENIX at RHIC/BNL [57] [58] to analyze the explicit data points for direct photons and provided the most interesting results. Hence, electromagnetic probes are considered to be ideal probes for the detection and study of subsequent evolution of QGP.

The earlier observations of photon production from quark-gluon plasma at finite temperature have been performed in Ref. [59]-[63]. In 1-loop approximation, the photon emission rate from annihilation and Compton processes has been calculated by Ref. [3]. Further, Aurenche et al. [64]-[66] has shown photon production rate in the QGP upto 2-loop level and found that emission rate are considerably same size or larger than the earlier lowest order results. A new annihilation with scattering (aws), higher order process was found to dominate the photon production rate from quark matter at high photon energies. With these informations, we focus on the measurement of photon emission which provide a good opportunity to study the early evolution of fireball.

In this work, we use the thermal mass formalism and the corresponding thermal Hamiltonian in the literature leading to the following choice for the confining/de-confining potential [67]-[69]. The phenomenological model is used as quasiparticle in which mass is dependent on temperature and parametrization factors [70]-[72]. This effective mass generated due to thermal interactions between quarks and gluons and shows well behaviour above and around critical temperature. The "Thermal-Hamiltonian” for the QGP is given as:

$$
H(q, T)=\left[q^{2}+m_{q}^{2}(T)\right]^{1 / 2} \equiv q+m_{q}^{2}(T) / 2 q
$$

The above result is taken in the large $q$ limit or expressed as,

$$
=q+m_{0}^{2} / 2 q-\left\{m_{0}^{2}-m_{q}^{2}(T)\right\} / 2 q,
$$

where,

$$
m_{q}^{2}(T)=\gamma_{g, q} g^{2}(q) T^{2} .
$$

with $q$ is the quarks (gluons) momentum, $m_{0}$ is the rest mass of the quark, $T$ is the temperature and $g(q)$ is first order QCD running coupling constant. $\gamma_{g, q}$ is the phenomenological parameter used to take care the hydrodynamical aspects of the hot QGP droplet. We fix $\gamma_{g}=6 \gamma_{q}$ or $8 \gamma_{q}$ and $\gamma_{q}=1 / 6$. All parameter values are taken by Ref. [73]-[75] in our calculation,

$$
g^{2}(q)=\frac{4}{3} \frac{12 \pi}{\left(33-2 n_{f}\right)} \frac{1}{\ln \left(1+q^{2} / \Lambda^{2}\right)},
$$

where $\Lambda$ is the QCD scale taken to be $150 \mathrm{MeV}, n_{f}$ is the number of flavor. Finally, we obtain the finite quark mass defined as [73]-[75]:

$$
m_{q}^{2}(T)=\frac{1}{6} \frac{N}{\ln \left[1+\frac{q^{2}}{\Lambda^{2}}\right]} T^{2}
$$

where $q=\left[\frac{\gamma N^{\frac{1}{3}} T^{2} \Lambda^{2}}{2}\right]^{\frac{1}{4}}$ known as minimum momentum cut off with $N=\frac{16 \pi}{\left[33-2 n_{f}\right]}$ and flow parameter is taken as $\gamma^{2}=2\left[\frac{1}{\gamma_{q}^{2}}+\frac{1}{\gamma_{g}^{2}}\right]$. The finite value of quark mass also removes the infrared divergence produced in photons production [76]-[78]. We compute the thermal photon emission from quark-gluon plasma of complete 
leading order (LO) results at temperatures $T=0.25 \mathrm{GeV}$ and $T=0.30 \mathrm{GeV}$ with parametrization factor for flavor 2 and 3 and compare the results with other work.

The paper is organized as follows. In Section 2 we outline the LO processes for photon spectra in QGP. Results are presented in Section 3. Finally in Section 4 we give the conclusion.

\section{Emission of Thermal Photon from QGP}

Various theoretical predictions have been proved, some of the surprises have been found in experimental results and indicate that RHIC and LHC is, indeed, probing a new development in the field of high energy physics. One loop and two loop order for complete calculation of photon production rate from QGP to order $\alpha_{s}$ have been considered in the Ref. [59]-[61] [64]-[66] [79] [80]. The relevant LO processes include different channels. For the present calculation, we use the parametrization of the rate given in Ref. [80].

The rate for photons of momentum $q$ is given by the expression [80] [81]

$$
\frac{\mathrm{d} N}{\mathrm{~d}^{4} x \mathrm{~d}^{3} q}=\frac{1}{(2 \pi)^{3}} A(q)\left[\ln \left(\frac{T}{m_{q}(T)}\right)+\frac{1}{2} \ln \left(\frac{2 E}{T}\right)+C_{t o t}\left(\frac{E}{T}\right)\right]
$$

with $E=q$ and $m_{q}^{2}(T)$ is the leading order large momentum limit of the thermal quark mass. The leading$\log$ coefficient $A(q)$ is given as

$$
A(q)=6 \alpha_{e} \sum_{f} e_{f}^{2} \frac{m_{q}^{2}(T)}{E} f_{D}(E)
$$

The summation is over the number of quark flavors and $e_{f}^{2}$ is their electric charge, $\alpha_{e}$ is the electromagnetic constant and mass of quark are taken by Ref. [73]-[75]. $f_{D}(E)$ is the fermi distribution function. The dependence on the specific photon production process is written in the term $C_{\text {tot }}\left(\frac{E}{T}\right)$,

$$
C_{\text {tot }}\left(\frac{E}{T}\right)=C_{2 \leftrightarrow 2}\left(\frac{E}{T}\right)+C_{\text {brems }}\left(\frac{E}{T}\right)+C_{\text {aws }}\left(\frac{E}{T}\right) \text {. }
$$

The non-logarithmic two-to-two contribution $C_{2 \leftrightarrow 2}$ for general $E / T$ and the rate of photon production by bremsstrahlung and annihilation with scattering are computed. This requires solving a non-trivial integral equation to determine this rate. The thermal corrections to the dispersion relations for incoming or outgoing particles can no longer be neglected in these $2 \leftrightarrow 2$ processes. The $C_{\text {tot }}\left(\frac{E}{T}\right)$ is the non-trivial function that can only be solved numerically. All results for $C_{\text {tot }}\left(\frac{E}{T}\right)$ are evaluated by Ref. [80] [81].

Then we study the total photon spectrum above critical temperature by integrating the total rate over the space-time history of the collision for all the LO processes after getting the temperature of the evolution from the model. We integrate rates at preferential temperatures, which are considered as temperature of transition to be completely hot phase. It is expressed as [75] [81]:

$$
\frac{\mathrm{d} N}{\mathrm{~d}^{2} q_{T} \mathrm{~d} y}=\int \mathrm{d}^{4} x\left(E \frac{\mathrm{d} N}{\mathrm{~d}^{3} q \mathrm{~d}^{4} x}\right)=Q \int_{\tau_{0}}^{\tau_{f}} \tau \mathrm{d} \tau \int \mathrm{d} y\left(E \frac{\mathrm{d} N}{\mathrm{~d}^{3} q \mathrm{~d}^{4} x}\right)
$$

where $\tau$ is time evolution determined with the temperature from initial to final state with the rapidity $y_{\text {пис }}= \pm 5.3$ corresponding to RHIC energy. $Q \sim 180 \mathrm{fm}^{2}$ is transverse cross section and $q_{T}$ is the photon transverse momentum. The quantity on the extreme R.H.S. is defined in the centre-of-mass system with the photon energy $E=q_{T} \cosh \left(y^{\prime}-y\right)$. Thus, with the values of rapidity and $q_{T}$, we get the total photon spectrum of LO processes. 


\section{Results}

The results are important concerning photon production as a signature for the creation of a quark-gluon plasma. We perform the calculation of LO processes for photon emission in the QGP which consist QCD Compton Scattering, $q \bar{q}$ annihilation, Bremsstrahlung (brems), and annihilation with scattering (aws) with suitable choice of parametrization factors in the quark mass at hot phase of QGP evolution with temperature $T=0.25 \mathrm{GeV}$ and $T=0.30 \mathrm{GeV}$ for flavor $n_{f}=2$ and 3 .

In Figure 1, we plot LO processes for photon emission rate at fixed temperature $T=0.25 \mathrm{GeV}$ for flavor $n_{f}=2$ and 3 with the effect of finite value of quark mass with the variation of the photon energy. At closer inspection, it turns out that the model calculation in the relevant range give the significant contribution and dominate over the results of Renk et al. [81]. The results show visible increment at temperature $T=0.25 \mathrm{GeV}$ of the QGP fireball for flavor $n_{f}=2$. We also show the result for flavor 3 and found that the strength of photon emission is enhanced for the large flavor numbers. The larger number of anticipating quarks bring more interactions, resulting in the enhancement of production rate. So with introducing the strange mass, photon emission increases. The increase in the emission rate is highly effected by temperature of the system, and it seems to be large near creation of quark matter that is considered to be exist at very hot temperature. We compare the outputs between our result and the standard result of Ref. [81]. Our results has visible incremental amount in LO production rate over the result of Ref. [81].

Now, we study the total photon spectrum over the space-time evolution of QGP. The results are shown in Figure 2. With the similar line of parameters, we show total emission rate as a function of transverse momentum at temperature $T=0.30 \mathrm{GeV}$ and compare the results produced by Ref. [81]. In the yield rate of two different quark flavors, the higher value of quark flavor $n_{f}=3$ has larger yield than lower value. It is due to the more interaction among the large number of constituent quarks. In the comparison, we find that the photon spectra of our model for quark flavor $n_{f}=2$ has visible improvement, indeed, enhacement over theoretical result of Ref. [81]. Thus, the consideration of thermal dependent quark mass has an important role in LO processes for photon measurements and does not invalidate the results of high energy heavy-ion collisions. This simple phenomenological model shows little improved result over the results of Ref. [81]. The figure also shows that production rate as function of transverse momentum have much enhancement over the production rate as function of photon energy.

\section{Conclusion}

The measurement of leading order processes for photon production in the QGP which consist QCD Compton Scattering, $q(\bar{q})$ annihilation, Bremsstrahlung, and annihilation with scattering provides a good opportunity to study the evolution of fireball in high-energy heavy-ion collisions. We have used the leading order photon

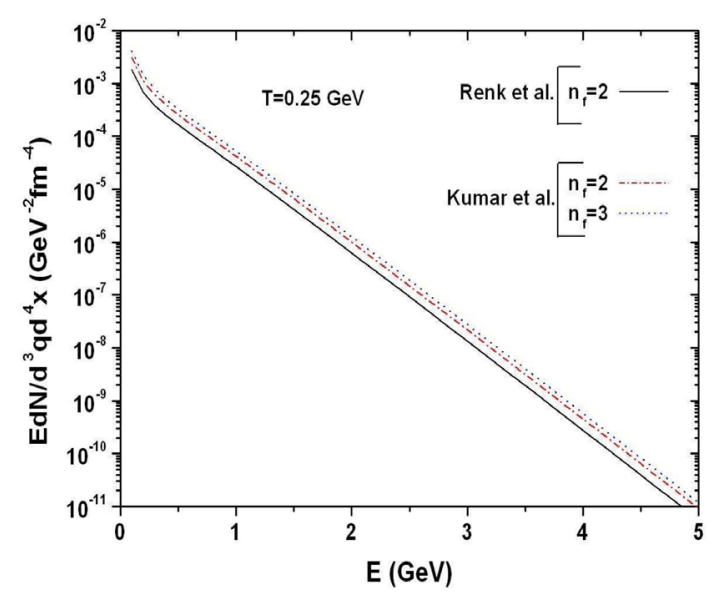

Figure 1. The photon spectra at thermal temperature $T=0.25 \mathrm{GeV}$ for $n_{f}=2$ and 3 and compared with other work. 


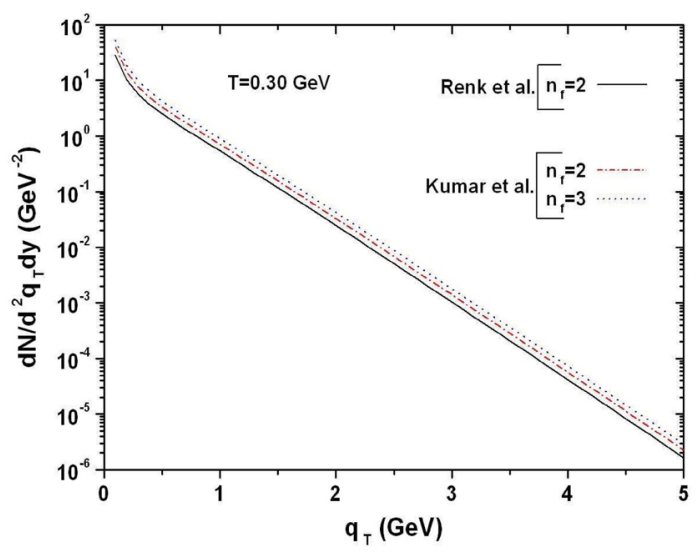

Figure 2. The total photon rate at thermal temperature $T=0.30 \mathrm{GeV}$ for $n_{f}=2$ and 3 and compared with other work.

emission rate from QGP, along with the estimates of the impact of our phenomenological model on this rate, in a simple model for the fireball evolution to calculate the resulting photon spectrum. The work on LO processes for thermal photons have discussed within a model with various sets of initial condition. This implies that the consideration of thermal dependent quark mass has important role in the photon measurements of the highenergy heavy-ion collisions. The QGP fireball with the model of parametrization factor give a significant contribution and improved the calculation of photon radiation for 2 and 3 flavor in high-energy heavy-ion collisions.

\section{Acknowledgements}

We thank V. Kumar for suggestions and discussions in preparing the manuscript.

\section{References}

[1] Hung, C.M. and Shuryak, E. (1998) Physics Review C, 57, 1891. http://dx.doi.org/10.1103/PhysRevC.57.1891

[2] Alam, J., Srivastava, D.K., Sinha, B. and Basu, D.N. (1993) Physics Review D, 48, 1117. http://dx.doi.org/10.1103/PhysRevD.48.1117

[3] Kapusta, J., Lichard, P. and Seibert, D. (1991) Physics Review D, 44, 2774-2788. http://dx.doi.org/10.1103/PhysRevD.44.2774

[4] Kapusta, J., Lichard, P. and Seibert, D. (1992) Nuclear Physics A, 544, 485-491. http://dx.doi.org/10.1016/0375-9474(92)90603-H

[5] Arsene, I., et al. (2005) Nuclear Physics A, 757, 1-27. http://dx.doi.org/10.1016/j.nuclphysa.2005.02.130

[6] Adcox, K., et al. (2005) Nuclear Physics A, 757, 184-283. http://dx.doi.org/10.1016/j.nuclphysa.2005.03.086

[7] Back, B.B., et al. (2005) Nuclear Physics A, 757, 28-101. http://dx.doi.org/10.1016/j.nuclphysa.2005.03.084

[8] Adams, J., et al. (2005) Nuclear Physics A, 757, 102-183. http://dx.doi.org/10.1016/j.nuclphysa.2005.03.085

[9] Adare, A., et al. (2010) Physics Review C, 81, Article ID: 034911. http://dx.doi.org/10.1103/PhysRevC.81.034911

[10] Adler, S.S., et al. (2007) Physics Review Letter, 98, Article ID: 012002. http://dx.doi.org/10.1103/PhysRevLett.98.012002

[11] Adare, A., et al. (2007) Physics Review Letter, 98, Article ID: 162301. http://dx.doi.org/10.1103/PhysRevLett.98.162301

[12] Adcox, K., et al. (2002) Physics Review Letter, 88, Article ID: 022301. http://dx.doi.org/10.1103/PhysRevLett.88.022301

[13] Adler, C., et al. (2002) Physics Review Letter, 89, Article ID: 092301. http://dx.doi.org/10.1103/PhysRevLett.89.092301

[14] Adler, S.S., Afanasiev, S., Aidala, C., et al. (2003) Physics Review Letter, 91, Article ID: 072301. http://dx.doi.org/10.1103/PhysRevLett.91.072301 
[15] Chujo, T. (2003) Nuclear Physics A, 715, 151c-160c. http://dx.doi.org/10.1016/S0375-9474(02)01423-9

[16] Adare, A., Afanasiev, S., Aidala, C., Ajitanand, N.N., Akiba, Y., Al-Bataineh, H., et al. (2010) Physics Review Letters, 104, Article ID 132301. http://dx.doi.org/10.1103/PhysRevLett.104.132301

[17] Büsching, H.B. (2006) Nuclear Physics A, 774, 103-112. http://dx.doi.org/10.1016/j.nuclphysa.2006.06.033

[18] Braun-Munzinger, P., Specht, H.J. and Stocker, H. (1996) Nuclear Physics A, 610, xi-xii.

[19] Geiger, K. and Müller, B. (1992) Nuclear Physics B, 369, 600-654. http://dx.doi.org/10.1016/0550-3213(92)90280-O

[20] Srivastava, D.K. and Sinha, B. (1994) Physics Review Letter, 73, 2421. http://dx.doi.org/10.1103/PhysRevLett.73.2421

[21] Chatterjee, R., Frodermann, E.S., Heinz, U.W. and Srivastava, D.K. (2006) Physics Review Letter, 96, Article ID: 202302. http://dx.doi.org/10.1103/PhysRevLett.96.202302

[22] Jeon, S., Marian, J.J. and Sarcevic, I. (2003) Nuclear Physics A, 715, 795c-798c. http://dx.doi.org/10.1016/S0375-9474(02)01491-4

[23] Kolb, P.F., Sollfrank, J. and Heinz, U.W. (2000) Physics Review C, 62, Article ID: 054909. http://dx.doi.org/10.1103/PhysRevC.62.054909

[24] Yang, S., Guo, H., Zhao, E.G. and Lu, X.F. (2007) Chinese Physics Letters, 24, 3096. http://dx.doi.org/10.1088/0256-307X/24/11/022

[25] Liu, H., Hou, D.F. and Li, J.R. (2007) Chinese Physics Letter, 24, 1191. http://dx.doi.org/10.1088/0256-307X/24/5/018

[26] Shi, X.H., Ma, Y.G., Cai, X.Z., Chen, J.H., Ma, G.L. and Zhong, C. (2009) Chinese Physics Letter, 26, Article ID: 061202. http://dx.doi.org/10.1088/0256-307X/26/6/061202

[27] Xiang, W.C., Wan, R.Z. and Zhou, D.C. (2008) Chinese Physics Letter, 25, 3912. http://dx.doi.org/10.1088/0256-307X/25/11/021

[28] Chang, S., Liu, J.F. and Zhuang, P.F. (2008) Chinese Physics Letter, 25, 55. http://dx.doi.org/10.1088/0256-307X/25/1/016

[29] QM01 (2002) Nuclear Physics A, 698.

[30] Gyulassy, M. (2004) The QGP Discovered at RHIC.

[31] Shuryak, E. (2004) What RHIC Experiments and Theory Tell Us about Properties of Quark-Gluon Plasma?.

[32] Heinz, U. (2004) Thermalization at RHIC.

[33] Shuryak, E.V. (1978) Physics Letters B, 78, 150-153. http://dx.doi.org/10.1016/0370-2693(78)90370-2

[34] Srivastava, D.K., Gale, C. and Fries, R.J. (2003) Physics Review C, 67, Article ID: 034903. http://dx.doi.org/10.1103/PhysRevC.67.034903

[35] Turbide, S., Rapp, R. and Gale, C. (2004) Physics Review C, 69, Article ID: 014903. http://dx.doi.org/10.1103/PhysRevC.69.014903

[36] Kapusta, J., Mclerran L.D. and Srivastava, D.K. (1992) Physics Letters B, 283, 145-150. http://dx.doi.org/10.1016/0370-2693(92)91445-F

[37] Turbide, S., Gale, C., Jeon, S. and Moore, G.D. (2005) Physics Review C, 72, Article ID: 014906. http://dx.doi.org/10.1103/PhysRevC.72.014906

[38] Adams, J., Adler, C., Aggarwal, M.M., Ahammed, Z., Amonett, J., Anderson, B.D., et al. (2003) Physics Review Letter, 91, Article ID: 172302. http://dx.doi.org/10.1103/PhysRevLett.91.172302

[39] Adler, C., Ahammed, Z., Allgower, C., Amonett, J., Anderson, B.D., Anderson, M., et al. (2003) Physics Review Letter, 90, Article ID: 082302. http://dx.doi.org/10.1103/PhysRevLett.90.082302

[40] Renk, T. (2006) Physics Review C, 74, Article ID: 034906. http://dx.doi.org/10.1103/PhysRevC.74.034906

[41] Arleo, F., et al. (2004) Photon Physics in Heavy Ion Collisions at the LHC.

[42] Shuryak, E.V. (1980) Physics Report, 61, 71-158. http://dx.doi.org/10.1016/0370-1573(80)90105-2

[43] Kajantie, K. and Miettinen, H.I. (1981) Zeitschrift fuer Physik C Particles and Fields, 9, 341-345. http://dx.doi.org/10.1007/BF01548770

[44] Domikos, G. and Goldman, J. (1981) Physics Review D, 23, 203. http://dx.doi.org/10.1103/PhysRevD.23.203

[45] Mclerran, L.D. and Toimela, T. (1985) Physics Review D, 31, 545-563. http://dx.doi.org/10.1103/PhysRevD.31.545

[46] Gale, C. and Kapusta, J.I. (1991) Nuclear Physics B, 357, 65-89. http://dx.doi.org/10.1016/0550-3213(91)90459-B

[47] Peitzmann, T. and Thoma, M.H. (2002) Physics Reports, 364, 175-246. 
http://dx.doi.org/10.1016/S0370-1573(02)00012-1

[48] Cassing, W. and Bratkovskaya, E.L. (1999) Physics Report, 308, 65-233. http://dx.doi.org/10.1016/S0370-1573(98)00028-3

[49] Steffen, F.D. and Thoma, M.H. (2001) Physics Letter B, 510, 98-106. http://dx.doi.org/10.1016/S0370-2693(01)00525-1

[50] Traxler, C.T., Vija, H. and Thoma, M.H. (1995) Physics Letter B, 346, 329-334. http://dx.doi.org/10.1016/0370-2693(95)00004-5

[51] Traxler, C.T. and Thoma, M.H. (1996) Physics Review C, 53, 1348-1352.

[52] Gale, C., Turbide, S., Frodermann, E. and Heinz, U. (2008) Journal of Physics G: Nuclear and Particle Physics, 35, Article ID: 104119. http://dx.doi.org/10.1088/0954-3899/35/10/104119

[53] Dusling, K. and Zahed, I. (2010) Physics Review C, 82, Article ID: 054909.

[54] Aggarwal, M.M., Nilsson, P., Nishimura, S., Nomokonov, P., Nystrand, J., Obenshain, F.E., et al. (2000) Physics Review Letters, 85, 3595-3599. http://dx.doi.org/10.1103/PhysRevLett.85.3595

[55] Aggarwal, M.M., Ahammed, Z., Angelis, A.L.S., Antonenko, V., Arefiev, V., Astakhov, V., et al. (2004) Physics Review Letters, 93, Article ID: 022301.

[56] Albrecht, R., Antonenko, V., Awes, T.C., Barlag, C., Berger, F., Bloomer, M., et al. (1996) Physics Review Letter, 76, 3506-3509. http://dx.doi.org/10.1103/PhysRevLett.76.3506

[57] Adler, S.S., Afanasiev, S., Aidala, C., Ajitanand, N.N., Akiba, Y., Alexander, J., et al. (2005) Physics Review Letter, 94, Article ID: 232301. http://dx.doi.org/10.1103/PhysRevLett.94.232301

[58] Morrison, D.P., Akiba, Y., Alford, O., Allen, M., Allen, W., Alley, G., et al. (1998) Nuclear Physics A, 638, 565c-569c. http://dx.doi.org/10.1016/S0375-9474(98)00390-X

[59] Kapusta, J., Lichard, P. and Seibert, D. (1993) Physics Review D, 47, 4171. http://dx.doi.org/10.1103/PhysRevD.47.4171

[60] Baier, R., Nakkagawa, H., Neigawa, A. and Redlich, K. (1992) Zeitschrift fuer Physik C Particles and Fields, 53, 433438. http://dx.doi.org/10.1007/BF01625902

[61] Alam, J., Sarkar, S., Hatsuda, T., Nayak, T.K. and Sinha, B. (2001) Physics Review C, 63, Article ID: 021901.

[62] Wang, S.Y. and Boyanovsky, D. (2001) Physics Review D, 63, Article ID: 051702.

[63] Boyanovsky, D. and de Vega, H.J. (2003) Physics Review D, 68, Article ID: 065018.

[64] Aurenche, P., Elis, F., Kobes, R. and Zaraket, H. (1998) Physics Review D, 58, Article ID: 085003.

[65] Aurenche, P., Gelis, F. and Zaraket, H. (2000) Physics Review D, 61, Article ID: 116001. http://dx.doi.org/10.1103/PhysRevD.61.116001

[66] Aurenche, P., Gelis, F. and Zaraket, H. (2000) Physics Review D, 62, Article ID: 096012. http://dx.doi.org/10.1103/PhysRevD.62.096012

[67] Ramanathan, R., Mathur, Y.K., Gupta, K.K. and Jha, A.K. (2004) Physical Review C, 70, Article ID: 027903. http://dx.doi.org/10.1103/PhysRevC.70.027903

[68] Ramanathan, R., Gupta, K.K., Jha, A.K. and Singh, S.S. (2007) Pramana, 68, 757-768.

[69] Singh, S.S., Gosain, D.S., Kumar, Y. and Jha, A.K. (2010) Pramana, 74, 27-37. http://dx.doi.org/10.1007/s12043-010-0004-0

[70] Peshier, A., Kampfer, B., Pavlenko, O.P. and Soff, G. (1994) Physics Letters B, 337, 235-239. http://dx.doi.org/10.1016/0370-2693(94)90969-5

[71] Gorenstein, M.I. and Yang, S.N. (1995) Physics Review D, 52, 5206. http://dx.doi.org/10.1103/PhysRevD.52.5206

[72] Goloviznin, V. and Satz, H. (1993) Zeitschrift fuer Physik C Particles and Fields, 57, 671-675. http://dx.doi.org/10.1007/BF01561487

[73] Kumar, Y. and Singh, S.S. (2012) Canadian Journal of Physics, 90, 955-961. http://dx.doi.org/10.1139/p2012-089

[74] Singh, S.S. and Kumar, Y. (2013) Journal of Modern Physics, 4, 582-586. http://dx.doi.org/10.4236/jmp.2013.45082

[75] Kumar, Y. and Singh, S.S. (2013) ISRN High Energy Physics, 2013, Article ID: 156747. http://dx.doi.org/10.1155/2013/156747

[76] Pisarski, R.D. (1988) Nuclear Physics B, 309, 476-492. http://dx.doi.org/10.1016/0550-3213(88)90454-3

[77] Pisarski, R.D. (1989) Physics Review Letter, 63, 1129-1132. http://dx.doi.org/10.1103/PhysRevLett.63.1129

[78] Braaten, E. and Pisarski, R.D. (1990) Nuclear Physics B, 337, 569-634. 
http://dx.doi.org/10.1016/0550-3213(90)90508-B

[79] Arnold, P., Moore, G.D. and Yaffe, L.G. (2001) Journal of High Energy Physics, 0111, 057.

[80] Arnold, P., Moore, G.D. and Yaffe, L.G. (2001) Journal of High Energy Physics, 0112, 009.

[81] Renk, T. (2003) Physics Review C, 67, Article ID: 064901. 
Scientific Research Publishing (SCIRP) is one of the largest Open Access journal publishers. It is currently publishing more than 200 open access, online, peer-reviewed journals covering a wide range of academic disciplines. SCIRP serves the worldwide academic communities and contributes to the progress and application of science with its publication.

Other selected journals from SCIRP are listed as below. Submit your manuscript to us via either submit@scirp.org or Online Submission Portal.
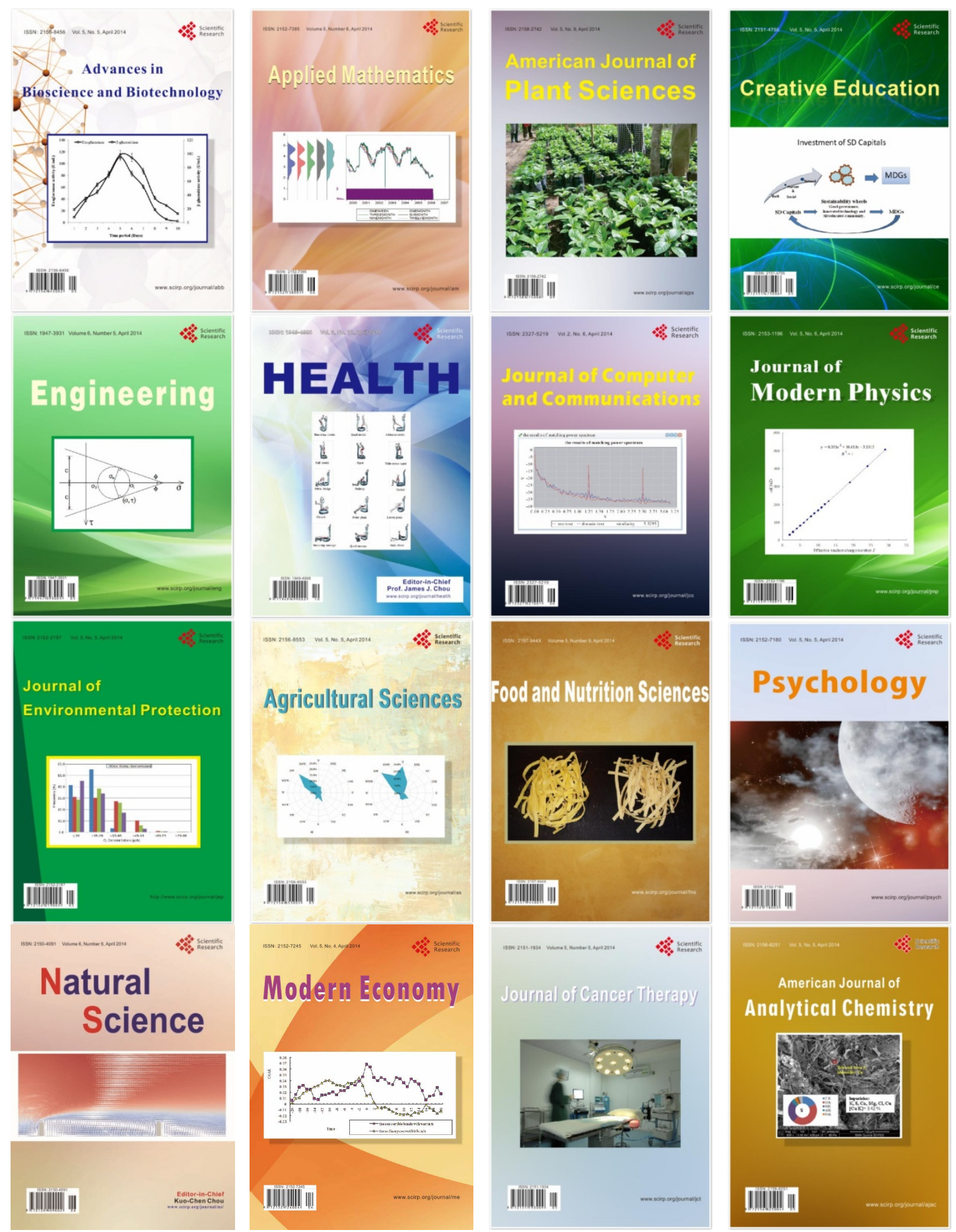